\title{
On the infinite product exponents of meromorphic modular forms for certain arithmetic groups
}

\author{
by
}

SoYoung Chor (Gyeongju) and Chang Heon Kim (Seoul)

1. Introduction. By using Ramanujan's theta operator, Bruinier, Kohnen and Ono [6] obtained formulae for the infinite product exponents of meromorphic modular forms for $S L_{2}(\mathbb{Z})$ which are determined by the divisors of the modular forms. Ahlgren [1] generalized this result to Hecke subgroups $\Gamma_{0}(p)$ of $S L_{2}(\mathbb{Z})$ with $p \in\{2,3,5,7,13\}$. Let $\Gamma_{0}^{+}(N)$ be the group generated by the Hecke subgroup $\Gamma_{0}(N)$ and the Fricke involution $W_{N}:=\left(\begin{array}{cc}0 & -1 \\ N & 0\end{array}\right)$. In this paper, we generalize the above-mentioned results to the groups $\Gamma_{0}^{+}(N)$ of genus zero, that is, to the groups $\Gamma_{0}^{+}(N)$, where $N$ lies in

$$
\Phi=\{1, \ldots, 21,23, \ldots, 27,29, \ldots, 32,35,36,39,41,47,49,50,59,71\} .
$$

As an application we reprove the formula for the number of representations of a given integer as a sum of four squares. The infinite product exponents of modular forms are also related to Borcherds product. Borcherds [2] showed that the infinite product exponents of certain modular functions for $S L_{2}(\mathbb{Z})$ come from Fourier coefficients of weakly holomorphic modular forms. On the other hand, in [15, Theorem 1] Zagier proved that the generating series for the traces of singular moduli of modular functions on $S L_{2}(\mathbb{Z})$ is a weakly holomorphic modular form of weight $3 / 2$ on $\Gamma_{0}(4)$. Here we give a connection between the traces of singular moduli of modular functions on $\Gamma_{0}^{+}(N)$ and the exponents of another modular function on $\Gamma_{0}^{+}(N)$. Moreover we express the Hurwitz-Kronecker class number via traces of singular moduli.

To state our results we require some notations. Throughout, we agree that $q=e^{2 \pi i z}$ for $z \in \mathfrak{H}$ (= complex upper half plane) and $N$ is a natural integer contained in $\Phi$, and denote the sum of divisors of $n$ by $\sigma(n)$. We

2010 Mathematics Subject Classification: Primary 11F03, 11F11; Secondary 11F37.

Key words and phrases: meromorphic modular forms, infinite product exponents.

Kim was supported by the Korea Research Foundation Grant funded by the Korean Government (MOEHRD) (KRF-2007-313-C00008). 
let $\mathfrak{H}^{*}=\mathfrak{H} \cup \mathbb{Q} \cup\{\infty\}$. Finally, for all positive integers $n$ let $j_{N, n}^{+}$be the modular function for $\Gamma_{0}^{+}(N)$ which is holomorphic on $\mathfrak{H}^{*}-\Gamma_{0}^{+}(N) \infty$ and has the Fourier expansion at $\infty$ of the form

$$
j_{N, n}^{+}(z)=\frac{1}{q^{n}}+\sum_{m=1}^{\infty} a_{j_{N, n}^{+}}(m) q^{m} .
$$

Let $h_{s}\left(\right.$ resp. $\left.\hbar_{s}\right)$ denote the width of a cusp $s$ in $\Gamma_{0}(N)$ (resp. $\left.\Gamma_{0}^{+}(N)\right)$. Let $\gamma$ be an element of $S L_{2}(\mathbb{Z})$ such that $\gamma \infty=s$. There exists a positive integer $w_{s}$ such that

$$
\left(\begin{array}{cc}
N & 0 \\
0 & 1
\end{array}\right) \gamma=\gamma^{\prime} U \quad \text { for some } \gamma^{\prime} \in S L_{2}(\mathbb{Z}) \text { and } U=\left(\begin{array}{cc}
x & y \\
0 & w_{s}
\end{array}\right) .
$$

We observe that the quantities $h_{s}, \hbar_{s}$ and $w_{s}$ are independent of the choice of $\gamma \in S L_{2}(\mathbb{Z})$ and only depend on the $\Gamma_{0}(N)$-class of $s$. More explicitly, if $s$ is a cusp represented by a rational number $a / c$ where $c$ is a divisor of $N$ and $a$ is coprime to $c$, then it is not difficult to verify that

$$
\begin{aligned}
& w_{s}=N / c, \quad \hbar_{s}=N /\left(c^{2}, N\right), \\
& h_{s}=\hbar_{s} \quad \text { unless } N=4 \text { and } c=2, \text { in which case } h_{s}=1 / 2 .
\end{aligned}
$$

Thus the quantity $h_{s}\left(1+N / w_{s}^{2}\right)$ which appears in Theorem 1.1 below depends only on the $\Gamma_{0}^{+}(N)$-class of $s$. Let $1 / e_{\tau}$ be the cardinality of the quotient $\Gamma_{0}^{+}(N)_{\tau} /\{ \pm 1\}$ for each $\tau \in \mathfrak{H}$, where $\Gamma_{0}^{+}(N)_{\tau}$ is the stabilizer of $\tau$ in $\Gamma_{0}^{+}(N)$. Let $\pi: \mathfrak{H}^{*} \rightarrow \Gamma_{0}^{+}(N) \backslash \mathfrak{H}^{*}$ be the canonical quotient map. With these notations we can state the first main result.

THEOREM 1.1. Suppose that $f=\sum_{n=h}^{\infty} a_{f}(n) q^{n}$ is a meromorphic modular form of weight $k$ for $\Gamma_{0}^{+}(N)$, normalized so that $a_{f}(h)=1$. Let

$$
f=q^{h} \prod_{n=1}^{\infty}\left(1-q^{n}\right)^{c_{f}(n)}
$$

for some complex numbers $c_{f}(n)$. Then for each integer $n \geq 1$ we have

$$
\begin{aligned}
\sum_{d \mid n} c_{f}(d) d= & k \sigma(n)+k N \sigma\left(\frac{n}{N}\right)+\sum_{\pi(\tau) \in \Gamma_{0}^{+}(N) \backslash \mathfrak{H}} e_{\tau} j_{N, n}^{+}(\tau) \operatorname{ord}_{\tau} f \\
& +\sum_{s \in S_{\Gamma_{0}^{+}(N)}-\{\infty\}} j_{N, n}^{+}(s)\left(\operatorname{ord}_{s} f-k h_{s}\left(\frac{1}{24}+\frac{N}{24 w_{s}^{2}}\right)\right),
\end{aligned}
$$

where $\operatorname{ord}_{\tau} f$ is the standard order of vanishing of $f, \operatorname{ord}_{s} f$ is the order of vanishing of $f$ at $s$, and $S_{\Gamma_{0}^{+}(N)}$ is the set of $\Gamma_{0}^{+}(N)$-inequivalent cusps.

Remark 1.2. (i) Suppose that the genus of $\Gamma_{0}(N)$ is zero. The same formula holds when $\Gamma_{0}^{+}(N)$ (respectively, $j_{N, n}^{+}$) is replaced by $\Gamma_{0}(N)$ (re- 
spectively, $\left.j_{N, n}\right)$ in Theorem 1.1. where $j_{N, n}$ is the modular function for $\Gamma_{0}(N)$ which is holomorphic on $\mathfrak{H}^{*}-\Gamma_{0}(N) \infty$ and has the Fourier expansion at $\infty$ of the form

$$
j_{N, n}(z)=\frac{1}{q^{n}}+\sum_{m=1}^{\infty} a_{j_{N, n}}(m) q^{m} .
$$

(ii) We mention that Choi [7] obtained another formula for the infinite product exponents of modular forms on $\Gamma_{0}(N)$ when $N$ is square free.

Let $\Theta$ be the Jacobi theta function:

$$
\Theta(z)=\sum_{n \in \mathbb{Z}} q^{n^{2}}:=\sum_{n=0}^{\infty} r_{1}(n) q^{n} .
$$

The number $r_{k}(n)$ of representations of an integer $n$ as a sum of $k$ squares is the coefficient of $q^{n}$ in the modular form $\Theta^{k}$. As an application of Theorem 1.1 we derive a formula for the number $r_{4}(n)$.

TheOREM 1.3. For each integer $n \geq 1$ we have

$$
r_{4}(n)=8 \sigma(n)-32 \sigma(n / 4),
$$

where $\sigma(n / 4)=0$ if 4 does not divide $n$.

We note that there are alternative proofs of this theorem. See [9, Theorem 386], [5, Proposition 11], [10, Theorem 5.33] and [13, Exercise III.5.2].

Let $d$ denote a positive integer congruent to 0 or 3 modulo 4 . We denote by $\mathcal{Q}_{d}$ the set of positive definite binary quadratic forms

$$
Q=[a, b, c]=a X^{2}+b X Y+c Y^{2} \quad(a, b, c \in \mathbb{Z})
$$

of discriminant $-d$, with the usual action of the modular group $\Gamma(1)=$ $S L_{2}(\mathbb{Z})$. To each $Q \in \mathcal{Q}_{d}$, we associate its unique root $\alpha_{Q} \in \mathfrak{H}$. Let $j(z)$ be the elliptic modular invariant defined on $\mathfrak{H}$ with a Fourier expansion

$$
j(z)=q^{-1}+744+196884 q+\cdots .
$$

The Hurwitz-Kronecker class number $H(d)$ and the trace $\mathbf{t}_{J}(d)$ for $J(z)=$ $j(z)-744$ are defined as

$$
H(d)=\sum_{Q \in \mathcal{Q}_{d} / \Gamma(1)} e_{\alpha_{Q}}, \quad \mathbf{t}_{J}(d)=\sum_{Q \in \mathcal{Q}_{d} / \Gamma(1)} e_{\alpha_{Q}} J\left(\alpha_{Q}\right) .
$$

Let $M_{1 / 2}^{!}$be the vector space of weakly holomorphic modular forms (that is, meromorphic with poles only at the cusps) of weight $1 / 2$ for $\Gamma_{0}(4)$ whose Fourier coefficients satisfy the Kohnen's "plus space" condition (i.e. the $n$th coefficients vanish unless $n \equiv 0$ or 1 modulo 4 ). For each nonnegative integer $d \equiv 0,3(\bmod 4)$ there is a unique modular form $f_{d} \in M_{1 / 2}^{!}$having a Fourier 
development of the form

$$
f_{d}(z)=q^{-d}+\sum_{D>0} A(D, d) q^{D} .
$$

We define a function $\mathcal{H}_{d}(X)$ by

$$
\mathcal{H}_{d}(X)=\prod_{Q \in \mathcal{Q}_{d} / \Gamma}\left(X-J\left(\alpha_{Q}\right)\right)^{e_{\alpha_{Q}}} .
$$

Then Borcherds' theorem [2] states that

$$
\mathcal{H}_{d}(J(z))=q^{-H(d)} \prod_{\nu=1}^{\infty}\left(1-q^{\nu}\right)^{A\left(\nu^{2}, d\right)} .
$$

Here we observe that the exponents of the modular function $\mathcal{H}_{d}(J(z))$ come from the coefficients of the weakly holomorphic modular forms $f_{d}$.

Let $d$ denote a positive integer such that $-d$ is congruent to a square modulo $4 N$. Let $\mathcal{Q}_{d, N}=\left\{[a, b, c] \in \mathcal{Q}_{d} \mid a \equiv 0(\bmod N)\right\}$ on which $\Gamma_{0}^{+}(N)$ acts. Let $f$ be a weakly holomorphic modular function for $\Gamma_{0}^{+}(N)$. We define the class number $H_{N}^{+}(d)$ and the trace $\mathbf{t}_{f}^{+}(d)$ by

$$
H_{N}^{+}(d)=\sum_{Q \in \mathcal{Q}_{d, N} / \Gamma_{0}^{+}(N)} e_{\alpha_{Q}}, \quad \mathbf{t}_{f}^{+}(d)=\sum_{Q \in \mathcal{Q}_{d, N} / \Gamma_{0}^{+}(N)} e_{\alpha_{Q}} f\left(\alpha_{Q}\right) .
$$

The higher level analogue of (3) was obtained in [11 by means of Borcherds lifting [3] when the level is prime. More generally, using Bruinier and Funke's work [4] on traces of singular moduli we derive the following result.

THEOREM 1.4. Let $N$ be a positive integer for which $\Gamma_{0}^{+}(N)$ is of genus zero. Let $j_{N}^{+}$be the corresponding Hauptmodul. For each cusp $s$ in $S_{N}:=$ $S_{\Gamma_{0}(N)}-\{\infty, 0\}$, define $k_{s}=(c, N / c)$ if $s$ is represented by a rational number $a / c$ such that $c$ is a divisor of $N$ and $a$ is coprime to $c$. Then for each positive integer $d$ such that $-d$ is congruent to a square modulo $4 N$ we have the following infinite product expansion:

$$
\begin{array}{r}
\prod_{s \in S_{N}}\left(j_{N}^{+}(z)-j_{N}^{+}(s)\right)^{-\frac{1}{2} k_{s} H\left(d / k_{s}^{2}\right)} \prod_{Q \in \mathcal{Q}_{d, N} / \Gamma_{0}^{+}(N)}\left(j_{N}^{+}(z)-j_{N}^{+}\left(\alpha_{Q}\right)\right)^{e_{\alpha}} \\
=q^{-H_{N}^{+}(d)+\sum_{s \in S_{N}} \frac{1}{2} k_{s} H\left(d / k_{s}^{2}\right)} \prod_{\nu=1}^{\infty}\left(1-q^{\nu}\right)^{-B\left(\nu^{2}, d\right)}
\end{array}
$$

where the class number $H\left(d / k_{s}^{2}\right)$ is defined to be zero if $k_{s}^{2} \nmid d$, and $B\left(\nu^{2}, d\right)$ is the coefficient of $q^{d}$ in a certain weakly holomorphic modular form of weight $3 / 2$ for $\Gamma_{0}(4 N)$. 
REMARK 1.5. For each $N \in \Phi$, we set

$$
\mathcal{H}_{d}^{j_{N}^{+}}(X)=\prod_{Q \in \mathcal{Q}_{d, N} / \Gamma_{0}^{+}(N)}\left(X-j_{N}^{+}\left(\alpha_{Q}\right)\right)^{e_{\alpha_{Q}}}
$$

and take

$$
f=\mathcal{H}_{d}^{j_{N}^{+}}\left(j_{N}^{+}(z)\right)=\prod_{Q \in \mathcal{Q}_{d, N} / \Gamma_{0}^{+}(N)}\left(j_{N}^{+}(z)-j_{N}^{+}\left(\alpha_{Q}\right)\right)^{e_{\alpha_{Q}}}
$$

so that $f$ (or some power of $f$ ) is a meromorphic modular form of weight 0 for $\Gamma_{0}^{+}(N)$. If we apply Theorem 1.1 to $f$ (or some power of $f$ ), then the exponent $c_{f}(n)$ satisfies the following identity:

$$
\mathbf{t}_{j_{N, n}^{+}}^{+}(d)=\sum_{\nu \mid n} c_{f}(\nu) \nu
$$

Let $N$ be a prime power and assume that the discriminant $d$ satisfies the Heegner condition (i.e. if we write $d=d_{K} \cdot c^{2}$, then $\left.(c, N)=1\right)$. In this case we note that $\left(k_{s}, N\right)>1$ for $s \in S_{N}$. Thus $H\left(d / k_{s}^{2}\right)=0$ and the product identity reduces to a simpler form

$$
f=\mathcal{H}_{d}^{j_{N}^{+}}\left(j_{N}^{+}(z)\right)=q^{-H_{N}^{+}(d)} \prod_{\nu=1}^{\infty}\left(1-q^{\nu}\right)^{-B\left(\nu^{2}, d\right)},
$$

so that the exponent of $f=\mathcal{H}_{d}^{j_{N}^{+}}\left(j_{N}^{+}(z)\right)$ arises from weakly holomorphic modular forms.

In Theorem 1.4, if $N=4$ and the discriminant is a multiple of 4 , we can get more information on the exponent, as shown in Lemma 3.4, which enables us to express the Hurwitz-Kronecker class numbers via traces of singular moduli, as in the following theorem.

THEOREM 1.6.

$H(d)$

$$
= \begin{cases}-\frac{1}{24 \sigma(n)} \mathbf{t}_{j_{4, n}^{+}}^{+}(4 d) & \text { if } n \text { is odd, } \\ -\frac{1}{24(\sigma(n)-4 \sigma(n / 2)+4 \sigma(n / 4))}\left(\mathbf{t}_{j_{4, n}^{+}}^{+}(4 d)-2 \mathbf{t}_{n / 2}(d)\right) & \text { if } n \text { is even. }\end{cases}
$$

Here $\mathbf{t}_{n / 2}(d)$ stands for $\mathbf{t}_{j_{1, n / 2}^{+}}^{+}(d)$.

We remark that Theorem 1.6 is an extension of [8, Theorem 1.2], but the style of the proof is different. 
2. Proofs of Theorems 1.1 and 1.3 . We denote by $E_{2}$ the weight two Eisenstein series

$$
E_{2}(z):=1-24 \sum_{n=1}^{\infty} \sigma(n) q^{n}
$$

We define the usual slash operator $\left.f\right|_{k} \gamma$ by

$$
\left(\left.f\right|_{k} \gamma\right)(z)=(\operatorname{det} \gamma)^{k / 2}(c z+d)^{-k} f(\gamma z),
$$

where $\gamma=\left(\begin{array}{ll}a & b \\ c & d\end{array}\right) \in G L_{2}^{+}(\mathbb{Q})$ and $\gamma z=\frac{a z+b}{c z+d}$. The action of Ramanujan's theta-operator is defined by

$$
\theta\left(\sum_{n=n_{0}}^{\infty} a(n) q^{n}\right):=\sum_{n=n_{0}}^{\infty} n a(n) q^{n} .
$$

We construct a modular form that will be important in this paper.

Lemma 2.1. Let $N$ be a positive integer and suppose that $f$ is a meromorphic modular form of weight $k$ for $\Gamma_{0}^{+}(N)$. Then

$$
F:=\frac{\theta f}{f}-\frac{k\left(E_{2}(z)+N E_{2}(N z)\right)}{24}
$$

is a meromorphic modular form of weight 2 for $\Gamma_{0}^{+}(N)$.

Proof. Let $\gamma=\left(\begin{array}{ll}a & b \\ c & d\end{array}\right) \in \Gamma_{0}^{+}(N)$. Since $f(\gamma z)=(\operatorname{det} \gamma)^{-k / 2}(c z+d)^{k} f(z)$, we have

$$
\frac{\theta f}{f}(\gamma z)=(\operatorname{det} \gamma)^{-1}\left[(c z+d)^{2} \frac{\theta f}{f}(z)+\frac{k c}{2 \pi i}(c z+d)\right] .
$$

We note that the transformation formula for $E_{2}$ is given by

(7) $\quad E_{2}(\gamma z)=(c z+d)^{2} E_{2}(z)-\frac{6 c i}{\pi}(c z+d) \quad$ for $\gamma=\left(\begin{array}{ll}a & b \\ c & d\end{array}\right) \in S L_{2}(\mathbb{Z})$

and the function $E_{2}^{*}(z):=E_{2}(z)-3 /(\pi \operatorname{Im}(z))$ is $S L_{2}(\mathbb{Z})$-invariant under the action of the slash operator. Hence $E_{2}^{*}(z)+\left(\left.E_{2}^{*}\right|_{2} W_{N}\right)(z)=E_{2}(z)+$ $N E_{2}(N z)-6 /(\pi \operatorname{Im}(z))$ is $\Gamma_{0}^{+}(N)$-invariant and therefore for $\gamma \in \Gamma_{0}^{+}(N)$,

$$
\begin{aligned}
E_{2}(\gamma z) & +N E_{2}(N \gamma z) \\
= & (\operatorname{det} \gamma)^{-1}\left[(c z+d)^{2}\left(E_{2}(z)+N E_{2}(N z)\right)-\frac{12 c i}{\pi}(c z+d)\right] .
\end{aligned}
$$

Consequently, the two functional equations (6) and (8) show that

$$
F(\gamma z)=(\operatorname{det} \gamma)^{-1}(c z+d)^{2} F(z) \quad \text { for } \gamma=\left(\begin{array}{ll}
a & b \\
c & d
\end{array}\right) \in \Gamma_{0}^{+}(N) .
$$


Now we investigate the behavior of $F(z)$ at the cusp $s$. Let $\gamma=\left(\begin{array}{ll}a & b \\ c & d\end{array}\right) \in$ $S L_{2}(\mathbb{Z})$ with $\gamma \infty=s$. We recall from (1) that

$$
\left(\begin{array}{cc}
N & 0 \\
0 & 1
\end{array}\right) \gamma=\gamma^{\prime} U \quad \text { for some } \gamma^{\prime}=\left(\begin{array}{cc}
* & * \\
c^{\prime} & d^{\prime}
\end{array}\right) \in S L_{2}(\mathbb{Z}) \text { and } U=\left(\begin{array}{cc}
x & y \\
0 & w_{s}
\end{array}\right) \text {. }
$$

Then we have

$$
\left.F\right|_{2} \gamma=\frac{\theta\left(\left.f\right|_{k} \gamma\right)}{\left.f\right|_{k} \gamma}-\frac{k E_{2}(z)}{24}-\frac{k N E_{2}(U z)}{24 w_{s}^{2}},
$$

which means that $F(z)$ is holomorphic at the cusp $s$. Indeed, we see that

$$
\left.\frac{\theta f}{f}\right|_{2} \gamma=\frac{\theta\left(\left.f\right|_{k} \gamma\right)}{\left.f\right|_{k} \gamma}+\frac{k c}{2 \pi i(c z+d)}
$$

and

$$
\begin{aligned}
E_{2}(N \gamma z)=E_{2}\left(\gamma^{\prime} U z\right) & =\left(c^{\prime} U z+d^{\prime}\right)^{2} E_{2}(U z)-\frac{6 c^{\prime} i}{\pi}\left(c^{\prime} U z+d^{\prime}\right) \\
& =\frac{(c z+d)^{2} E_{2}(U z)}{w_{s}^{2}}-\frac{6 c^{\prime} i(c z+d)}{\pi w_{s}}
\end{aligned}
$$

since $c^{\prime} U z+d^{\prime}=(c z+d) / w_{s}$. These two identities, (7) and the fact that $c^{\prime} / w_{s}=c / N$ imply (9). Consequently, the lemma follows.

A meromorphic weight two modular form $G(z)$ for $\Gamma_{0}^{+}(N)$ has a Fourier expansion at each cusp $s \in S_{\Gamma_{0}^{+}(N)}$ of the form

$$
\left(\left.G\right|_{2} \gamma\right)(z)=\sum_{n \geq N_{0}} a_{n} q_{h_{s}}^{n}
$$

where $\gamma$ is an element of $S L_{2}(\mathbb{Z})$ such that $\gamma \infty=s$. As usual, adjoining the cusps to $\Gamma_{0}^{+}(N) \backslash \mathfrak{H}$ we obtain a compact Riemann surface $X_{0}^{+}(N)$. Then for the corresponding differential $\omega_{G}=G(z) d z$ on $X_{0}^{+}(N)$, using the canonical quotient map $\pi: \mathfrak{H}^{*} \rightarrow X_{0}^{+}(N)$ and the Residue Theorem we obtain

$$
\sum_{p \in X_{0}^{+}(N)} \operatorname{Res}_{p} \omega_{G}=0 .
$$

We note that for each $s \in S_{\Gamma_{0}^{+}(N)}$ and $\tau \in \mathfrak{H}$,

$$
\operatorname{Res}_{\pi(s)} \omega_{G}=\frac{h_{s}}{2 \pi i} a_{0}, \quad \operatorname{Res}_{\pi(\tau)} \omega_{G}=e_{\tau} \operatorname{Res}_{\tau} G(z) .
$$

We are ready to prove our first main theorem which gives a formula for the exponents in the infinite product expansion of any modular form for $\Gamma_{0}^{+}(N)$.

For each $n \in \mathbb{Z}_{>0}$ let $G_{n}(z)=j_{N, n}^{+}(z) F(z)$ and $\omega_{n}=G_{n}(z) d z$. Then $\omega_{n}$ is a differential on $X_{0}^{+}(N)$. We calculate the residue of $\omega_{n}$ at each point $\pi(\tau)$ for $\tau \in \mathfrak{H}^{*}$. Using [6. Proposition 2.1] and the definitions of $F(z)$ and $E_{2}(z)$ 
we obtain

$$
\begin{aligned}
& G_{n}(z)=j_{N, n}^{+}(z) F(z) \\
& =\left(\frac{1}{q^{n}}+O(q)\right) \\
& \quad \times\left(\operatorname{ord}_{\infty} f-\frac{k+k N}{24}+\sum_{m=1}^{\infty}\left(-\sum_{d \mid m} c_{f}(d) d+k \sigma(m)+k N \sigma\left(\frac{m}{N}\right)\right) q^{m}\right) \\
& =\text { negative powers of } q+\left(-\sum_{d \mid n} c_{f}(d) d+k \sigma(n)+k N \sigma\left(\frac{n}{N}\right)\right)
\end{aligned}
$$

+ higher terms in $q$,

which means

$$
\operatorname{Res}_{\pi(\infty)} \omega_{n}=\frac{1}{2 \pi i}\left(-\sum_{d \mid n} c_{f}(d) d+k \sigma(n)+k N \sigma\left(\frac{n}{N}\right)\right) .
$$

For a cusp $s \in S_{\Gamma_{0}^{+}(N)}-\{\infty\}$ and $\gamma \in S L_{2}(\mathbb{Z})$ with $\gamma \infty=s$, we let

$$
j_{N, n}^{+}(\gamma z)=\sum_{m=0}^{\infty} b_{n, s}(m) q_{h_{s}}^{m} .
$$

The constant term $j_{N, n}^{+}(s):=b_{n, s}(0)$ is independent of the choice of $\gamma$. Then we obtain

$$
\begin{aligned}
\left(\left.G_{n}\right|_{2} \gamma\right) & (z)=\left(\left.\left(j_{N, n}^{+} F\right)\right|_{2} \gamma\right)(z)=j_{N, n}^{+}(\gamma z)\left(\left.F\right|_{2} \gamma\right)(z) \\
= & \left(\sum_{m=0}^{\infty} b_{n, s}(m) q_{h_{s}}^{m}\right)\left(\frac{\theta\left(\left.f\right|_{k} \gamma\right)}{\left.f\right|_{k} \gamma}-\frac{k}{24} E_{2}(z)-\frac{k N}{24 w_{s}^{2}} E_{2}(U(z))\right) \\
= & j_{N, n}^{+}(s)\left(\frac{\operatorname{ord}_{s} f}{h_{s}}-\frac{k}{24}-\frac{k N}{24 w_{s}^{2}}\right)+\text { higher terms in } q_{h_{s}}
\end{aligned}
$$

which means

$$
\operatorname{Res}_{\pi(s)} \omega_{n}=\frac{h_{s}}{2 \pi i} j_{N, n}^{+}(s)\left(\frac{\operatorname{ord}_{s} f}{h_{s}}-\frac{k}{24}-\frac{k N}{24 w_{s}^{2}}\right) .
$$

Let $\tau \in \mathfrak{H}$. Since $j_{N, n}^{+}(z)$ and $k E_{2}(z)+N E_{2}(N z)$ is holomorphic at $\tau$ we obtain

$$
\operatorname{Res}_{\pi(\tau)} \omega_{n}=e_{\tau} \operatorname{Res}_{\tau} j_{N, n}^{+}(z) \frac{\theta f}{f}=e_{\tau} j_{N, n}^{+}(\tau) \frac{\operatorname{ord}_{\tau} f}{2 \pi i} .
$$

Consequently, the Residue Theorem implies Theorem 1.1.

Next, we start with the proof of Theorem 1.3. We need three identities related to $r_{4}(n)$. Combining the identities we will find our formula. It is well known that $\Theta^{4}$ is a modular form of weight 2 for $\Gamma_{0}(4)$. Moreover by $[12$, 
p. 133] it has the following Fourier expansions at the cusps 0 and $1 / 2$, with $\alpha=\left(\begin{array}{cc}0 & -1 \\ 1 & 0\end{array}\right)$ and $\beta=\left(\begin{array}{cc}-1 & 0 \\ -2 & -1\end{array}\right)$ :

$$
\text { at } s=0: \quad\left(\left.\Theta^{4}\right|_{2} \alpha\right)(z)=-1 / 4+\text { higher terms in } q_{4} \text {, }
$$

$$
\text { at } s=1 / 2: \quad\left(\left.\Theta^{4}\right|_{2} \beta\right)(z)=2^{4} q+2^{6} q^{3}+\text { higher terms in } q .
$$

As usual, adjoining the cusps to $\Gamma_{0}(N) \backslash \mathfrak{H}$ we obtain a compact Riemann surface $X_{0}(N)$. Applying the Residue Theorem to the differential form $j_{4, n} \Theta^{4}(z) d z$ on $X_{0}(N)$ and using (10) and (11) we obtain the first identity

$$
r_{4}(n)=j_{4, n}(0),
$$

where $j_{4, n}$ is the modular function defined in Remark 1.2 .

Now, $F(z):=\left(\theta \Theta^{4}\right) / \Theta^{4}-E_{2}(z) / 6$ is a modular form of weight 2 for $\Gamma_{0}(4)$. Applying the Residue Theorem to the differential form $j_{4, n} F(z) d z$ on $X_{0}(N)$ and using (10) and (11) we have the second identity

$$
\sum_{d \mid n} c_{\Theta^{4}}(d) d=4 \sigma(n)+\frac{5 j_{4, n}(1 / 2)}{6}-\frac{2 j_{4, n}(0)}{3},
$$

where $\Theta^{4}(z)=\prod_{n=1}^{\infty}\left(1-q^{n}\right)^{c_{\Theta^{4}}(n)}$. Lastly by Remark 1.2 we have the third identity

$$
\sum_{d \mid n} c_{\Theta^{4}}(d) d=2 \sigma(n)+8 \sigma\left(\frac{n}{4}\right)+\frac{5 j_{4, n}(1 / 2)}{6}-\frac{5 j_{4, n}(0)}{12} .
$$

Combining the three identities we obtain Theorem 1.3 .

3. Proofs of Theorems 1.4 and 1.6. According to Bruinier and Funke's result [4], the generating series for the traces $\mathbf{t}_{j_{N, n}^{+}}^{+}(d)$ is the holomorphic part of a harmonic weak Maass form of weight $3 / 2$. Furthermore in [8] it was shown that by adding a suitable linear combination of weight $3 / 2$ Eisenstein series, one can always obtain a generating series that is a weakly holomorphic modular form. More precisely, by making use of [8, Theorem $3.2]$ if we proceed as in [8, Section 4], then we can obtain the following lemma.

Lemma 3.1. For each cusp $s \in S_{N}=S_{\Gamma_{0}(N)}-\{\infty, 0\}$, let $k_{s}$ be the positive rational number defined in Theorem 1.4. Then

$$
\begin{aligned}
\mathfrak{G}_{n}:= & -\sum_{\nu \mid n} \nu q^{-\nu^{2}}+\left(\sigma(n)+N \sigma(n / N)-\frac{1}{24} \sum_{s \in S_{N}} j_{N, n}^{+}(s)\left(\hbar_{s}-k_{s}\right)\right) \\
& +\sum_{m>0}\left(\boldsymbol{t}_{j_{N, n}^{+}}^{+}(m)-\frac{1}{2} \sum_{s \in S_{N}} j_{N, n}^{+}(s) k_{s} H\left(m / k_{s}^{2}\right)\right) q^{m}
\end{aligned}
$$

is a weakly holomorphic modular form of weight $3 / 2$. 
For each positive integer $\nu$, we inductively define $g_{\nu^{2}}$ such that

$$
\mathfrak{G}_{n}=-\sum_{\nu \mid n} \nu g_{\nu^{2}}
$$

We denote by $B\left(\nu^{2}, d\right)$ the coefficient of $q^{d}$ in $g_{\nu^{2}}$. It then follows from 12 and the definition of $\mathfrak{G}_{n}$ that

$$
\mathbf{t}_{j_{N, n}^{+}}^{+}(d)=\frac{1}{2} \sum_{s \in S_{N}} j_{N, n}^{+}(s) k_{s} H\left(d / k_{s}^{2}\right)-\sum_{\nu \mid n} \nu B\left(\nu^{2}, d\right) .
$$

Let $z, \tau \in \mathfrak{H}$. It is well known [14] that

$$
j_{N}^{+}(z)-j_{N}^{+}(\tau)=q^{-1} \exp \left(-\sum_{n=1}^{\infty} j_{N, n}^{+}(\tau) \frac{q^{n}}{n}\right) \quad(\operatorname{Im}(z) \gg 0) .
$$

We observe that

$$
\begin{aligned}
& \prod_{Q \in \mathcal{Q}_{d, N} / \Gamma_{0}^{+}(N)}\left(j_{N}^{+}(z)-j_{N}^{+}\left(\alpha_{Q}\right)\right)^{e_{\alpha_{Q}}} \\
& =q^{-H_{N}^{+}(d)} \exp \left(-\sum_{n=1}^{\infty} \mathbf{t}_{j_{N, n}^{+}}^{+}(d) \frac{q^{n}}{n}\right)
\end{aligned}
$$

by (14) and definition of $H_{N}^{+}(d)$ and $\mathbf{t}_{j_{N, n}^{+}}^{+}(d)$

$=q^{-H_{N}^{+}(d)} \exp \left(-\sum_{n=1}^{\infty} \sum_{s \in S_{N}} \frac{1}{2} j_{N, n}^{+}(s) k_{s} H\left(d / k_{s}^{2}\right) \frac{q^{n}}{n}\right) \exp \left(\sum_{n=1}^{\infty} \sum_{\nu \mid n} \nu B\left(\nu^{2}, d\right) \frac{q^{n}}{n}\right)$

by (13).

LEMMA 3.2.

$$
\begin{aligned}
\exp \left(-\sum_{n=1}^{\infty} \sum_{s \in S_{N}} \frac{1}{2} j_{N, n}^{+}(s) k_{s} H\left(d / k_{s}^{2}\right) \frac{q^{n}}{n}\right) \\
=q^{\sum_{s \in S_{N}} \frac{1}{2} k_{s} H\left(d / k_{s}^{2}\right)} \prod_{s \in S_{N}}\left(j_{N}^{+}(z)-j_{N}^{+}(s)\right)^{\frac{1}{2} k_{s} H\left(d / k_{s}^{2}\right)} .
\end{aligned}
$$

Proof. Let $s \in S_{N}$ be a cusp represented by $a / c$, where $c$ is a divisor of $N$ and $a$ is coprime to $c$. Choose integers $b$ and $d$ such that $a d-b c=1$. Then $a / c=\left(\begin{array}{ll}a & b \\ c & d\end{array}\right) \infty$ with $\left(\begin{array}{ll}a & b \\ c & d\end{array}\right) \in S L_{2}(\mathbb{Z})$. In $(14)$ if we replace $\tau$ by $\frac{a \tau+b}{c \tau+d}$ and send $\tau \rightarrow i \infty$, then we come up with

$$
j_{N}^{+}(z)-j_{N}^{+}(s)=q^{-1} \exp \left(-\sum_{n=1}^{\infty} j_{N, n}^{+}(s) \frac{q^{n}}{n}\right),
$$

from which the lemma easily follows. 
LEMMA 3.3.

$$
\exp \left(\sum_{n=1}^{\infty} \sum_{\nu \mid n} \nu B\left(\nu^{2}, d\right) \frac{q^{n}}{n}\right)=\prod_{\nu=1}^{\infty}\left(1-q^{\nu}\right)^{-B\left(\nu^{2}, d\right) .}
$$

Proof. Indeed,

$$
\begin{aligned}
\exp \left(\sum_{n=1}^{\infty} \sum_{\nu \mid n} \nu B\left(\nu^{2}, d\right) \frac{q^{n}}{n}\right) \\
=\exp \left(\sum_{n=1}^{\infty} \sum_{\nu=1}^{\infty} \nu B\left(\nu^{2}, d\right) \frac{q^{\nu n}}{\nu n}\right) \\
=\exp \left(\sum_{\nu=1}^{\infty}\left(-B\left(\nu^{2}, d\right) \sum_{n=1}^{\infty} \frac{-\left(q^{\nu}\right)^{n}}{n}\right)\right) \\
=\exp \left(\sum_{\nu=1}^{\infty} \log \left(1-q^{\nu}\right)^{-B\left(\nu^{2}, d\right)}\right)=\prod_{\nu=1}^{\infty}\left(1-q^{\nu}\right)^{-B\left(\nu^{2}, d\right)}
\end{aligned}
$$

Now Theorem 1.4 immediately follows from 15 and Lemmas 3.2 and 3.3. Next, to prove Theorem 1.6 we need the following lemma.

Lemma 3.4. Let d be a positive integer congruent to 0,1 modulo 4. Then

$$
\begin{aligned}
\mathcal{H}_{d}(J(2 z)) & =q^{-2 H(d)} \prod_{\nu=1}^{\infty}\left(1-q^{2 \nu}\right)^{A\left(\nu^{2}, d\right)} \\
& =\left(j_{4}^{+}(z)-j_{4}^{+}(1 / 2)\right)^{-H(d)} \mathcal{H}_{4 d}^{j_{4}^{+}}\left(j_{4}^{+}(z)\right) .
\end{aligned}
$$

Proof. The first equality follows from (3). For the second equality we note that $J(2 z)$ is a modular function for $\Gamma_{0}^{+}(4)$. Now we investigate the poles and zeros of the modular function $\mathcal{H}_{d}(J(2 z))$ on the modular curve $\Gamma_{0}^{+}(4) \backslash \mathfrak{H}^{*}$. There are two inequivalent cusps $\infty$ and $1 / 2$ under $\Gamma_{0}^{+}(4)$. Then $\mathcal{H}_{d}(J(2 z))$ has a pole of order $H(d)$ (resp. $2 H(d)$ ) at the cusp $1 / 2($ resp. $\infty$ ). And it has a usual simple zero at $\alpha_{Q}$ for each $Q=[4 a, 2 b, c] \in \mathcal{Q}_{4 d, 4} / \Gamma_{0}^{+}(4)$. Since our modular curve $\Gamma_{0}^{+}(4) \backslash \mathfrak{H}^{*}$ has genus zero, there is a unique rational function in $j_{4}^{+}$with prescribed zeros and poles. Now the assertion is immediate.

Taking the logarithmic derivative of (17) we come up with

$$
\begin{aligned}
& \sum_{Q \in \mathcal{Q}_{d} / \Gamma(1)} e_{\alpha_{Q}} \frac{\theta(J(2 z))}{J(2 z)-J\left(\alpha_{Q}\right)} \\
& \quad=-H(d) \frac{\theta\left(j_{4}^{+}(z)\right)}{j_{4}^{+}(z)-j_{4}^{+}(1 / 2)}+\sum_{Q \in \mathcal{Q}_{4 d, 4} / \Gamma_{0}^{+}(4)} e_{\alpha_{Q}} \frac{\theta\left(j_{4}^{+}(z)\right)}{j_{4}^{+}(z)-j_{4}^{+}\left(\alpha_{Q}\right)} .
\end{aligned}
$$


This combined with the logarithmic derivatives of (14) and (16) yields

$$
-2 \sum_{m \geq 1} \mathbf{t}_{m}(d) q^{2 m}=H(d) \sum_{n \geq 1} j_{4, n}^{+}(1 / 2) q^{n}-\sum_{n \geq 1} \mathbf{t}_{j_{4, n}^{+}}^{+}(4 d) q^{n} .
$$

Lemma 3.5. $j_{4, n}^{+}(1 / 2)=-24(\sigma(n)-4 \sigma(n / 2)+4 \sigma(n / 4))$ where $\sigma(x)$ is defined to be zero if $x \notin \mathbb{Z}$.

Proof. We recall that

$$
-\frac{\theta\left(j_{4}^{+}(z)\right)}{j_{4}^{+}(z)-j_{4}^{+}(1 / 2)}=1+\sum_{n=1}^{\infty} j_{4, n}^{+}(1 / 2) q^{n} .
$$

We observe that the left hand side of $(19)$ belongs to the space $M_{2}\left(\Gamma_{0}^{+}(4)\right)$. Note that $M_{2}\left(\Gamma_{0}(4)\right)$ is two-dimensional and spanned by $E_{2}^{(2)}(z)$ and $E_{2}^{(2)}(2 z)$ where $E_{2}^{(2)}(z)=E_{2}(z)-2 E_{2}(2 z)$. We see that

$$
E_{2}^{(2)}(z)+\left.E_{2}^{(2)}(z)\right|_{2} W_{4}=E_{2}^{(2)}(z)-2 E_{2}^{(2)}(2 z)=E_{2}(z)-4 E_{2}(2 z)+4 E_{2}(4 z)
$$

is invariant under the action of $W_{4}$ and spans the space $M_{2}\left(\Gamma_{0}^{+}(4)\right)$. Here the first equality of the above identity follows from (7). The left hand side of $(19)$ is then expressed as

$$
-\frac{\theta\left(j_{4}^{+}(z)\right)}{j_{4}^{+}(z)-j_{4}^{+}(1 / 2)}=E_{2}(z)-4 E_{2}(2 z)+4 E_{2}(4 z) .
$$

Now 19. combined with 20 yields the identity

$$
1+\sum_{n=1}^{\infty} j_{4, n}^{+}(1 / 2) q^{n}=E_{2}(z)-4 E_{2}(2 z)+4 E_{2}(4 z),
$$

from which the assertion is immediate.

Now 18 together with Lemma 3.5 yields Theorem 1.6 .

4. Example. Let $\eta(z)$ be the Dedekind eta-function defined by

$$
\eta(z)=q^{1 / 24} \prod_{n=1}^{\infty}\left(1-q^{n}\right)
$$

and let

$$
\begin{aligned}
j_{4}^{+}(z)= & \left(\frac{\eta(z)}{\eta(4 z)}\right)^{8}+8+4^{4}\left(\frac{\eta(4 z)}{\eta(z)}\right)^{8} \\
= & 1 / q+276 q+2048 q^{2}+11202 q^{3}+49152 q^{4} \\
& +184024 q^{5}+614400 q^{6}+1881471 q^{7}+\cdots
\end{aligned}
$$

be the Hauptmodul for $\Gamma_{0}^{+}(4)$. In Table 1 we shall compute the values of the class numbers $H(d)$ and the traces $\mathbf{t}(d), \mathbf{t}_{j_{4, n}^{+}}^{+}(d)$ for $1 \leq n \leq 3$. 
Table 1. Values of class numbers and traces

\begin{tabular}{ccc|ccccc}
\hline$d$ & $H(d)$ & $\mathbf{t}(d)$ & $d$ & $\mathcal{Q}_{d, 4} / \Gamma_{0}^{+}(4)$ & $\mathbf{t}_{j_{4}^{+}}^{+}(d)$ & $\mathbf{t}_{j_{4,2}^{+}}^{+}(d)$ & $\mathbf{t}_{j_{4,3}^{+}}^{+}(d)$ \\
\hline \multirow{3}{*}{3} & \multirow{2}{*}{$1 / 3$} & -248 & 12 & $\{[4,2,1]\}$ & -8 & -488 & -32 \\
& & & 15 & $\{[4,-1,1],[8,7,2]\}$ & -1 & -1 & -13114 \\
\multirow{2}{*}{4} & \multirow{2}{*}{$1 / 2$} & \multirow{2}{*}{492} & 16 & $\{[4,0,1],[4,-4,2]\}$ & -12 & 996 & -48 \\
\hline
\end{tabular}

By Lemma 3.1, we have weakly holomorphic modular forms

$$
\begin{aligned}
\mathfrak{G}_{n}= & -\sum_{\nu \mid n} \nu q^{-\nu^{2}}+\sigma(n)+4 \sigma(n / 4)-\frac{1}{24} j_{4, n}^{+}(1 / 2)(1-2) \\
& +\sum_{m>0}\left(\mathbf{t}_{j_{4, n}^{+}}^{+}(m)-j_{4, n}^{+}(1 / 2) H(m / 4)\right) q^{m} .
\end{aligned}
$$

And by Lemma 3.5 we compute that $j_{4,1}^{+}(1 / 2)=-24, j_{4,2}^{+}(1 / 2)=24$, $j_{4,3}^{+}(1 / 2)=-96$, etc. Utilizing the cusp values of $j_{4, n}^{+}$and Table 1 we have

$$
\begin{aligned}
& \mathfrak{G}_{1}=-q^{-1}+0-23 q^{7}-q^{15}+\cdots, \\
& \mathfrak{G}_{2}=-2 q^{-4}-q^{-1}+4-23 q^{7}-496 q^{12}-q^{15}+984 q^{16}+\cdots, \\
& \mathfrak{G}_{3}=-3 q^{-9}-q^{-1}+0+733 q^{7}-13114 q^{15}+\cdots, \text { etc. }
\end{aligned}
$$

from which it follows that

$$
\begin{aligned}
& g_{1}=q^{-1}+0+23 q^{7}+q^{15}+\cdots, \\
& g_{4}=q^{-4}-2+248 q^{12}-492 q^{16}+\cdots, \\
& g_{9}=q^{-9}+0-252 q^{7}+4371 q^{15}+\cdots, \text { etc. }
\end{aligned}
$$

Thus Theorem 1.4 yields the following product identities.

If $d=7$,

$$
j_{4}^{+}(z)+23=q^{-1}(1-q)^{-23}\left(1-q^{2}\right)^{0}\left(1-q^{3}\right)^{252} \cdots .
$$

If $d=12$,

$$
\left(j_{4}^{+}(z)+24\right)^{-1 / 3}\left(j_{4}^{+}(z)+8\right)=q^{-1+1 / 3}(1-q)^{0}\left(1-q^{2}\right)^{-248}\left(1-q^{3}\right)^{0} \cdots .
$$

From Table 1, we can check that

$$
-\frac{1}{24} \mathbf{t}_{j_{4}^{+}}^{+}(4 d)=-\frac{1}{96} \mathbf{t}_{j_{4,3}^{+}}^{+}(4 d)=H(d)
$$

and

$$
\frac{1}{24}\left(\mathbf{t}_{j_{4,2}^{+}}^{+}(4 d)-2 \mathbf{t}(d)\right)=H(d)
$$

as desired in Theorem 1.6 . 
Moreover in (17) if we put $d=3$, we obtain the following product identity by making use of Table 1, (2) and [15]:

$$
\begin{aligned}
\left(j_{4}^{+}(z)+24\right)^{-H(3)} \prod_{Q \in \mathcal{Q}_{12,4} / \Gamma_{0}^{+}(4)}\left(j_{4}^{+}(z)-j_{4}^{+}\left(\alpha_{Q}\right)\right)^{e_{\alpha}} & \\
= & q^{-2 H(3)} \prod_{\nu=1}^{\infty}\left(1-q^{2 \nu}\right)^{A\left(\nu^{2}, 3\right)}=\mathcal{H}_{3}(J(2 z)),
\end{aligned}
$$

which reads

$$
\begin{aligned}
\left(j_{4}^{+}(z)\right. & +24)^{-1 / 3}\left(j_{4}^{+}(z)+8\right) \\
& =q^{-2 / 3}\left(1-q^{2}\right)^{-248}\left(1-q^{4}\right)^{26752}\left(1-q^{6}\right)^{-4096248}=(J(2 z))^{1 / 3} .
\end{aligned}
$$

Acknowledgments. We would like to thank KIAS (Korea Institute for Advanced Study) for its hospitality. We also thank the referee for valuable comments.

\section{References}

[1] S. Ahlgren, The theta-operator and the divisors of modular forms on genus zero subgroups, Math. Res. Lett. 10 (2003), 787-798.

[2] R. Borcherds, Automorphic forms on $\mathrm{O}_{s+2,2}(\mathbb{R})$ and infinite products, Invent. Math. 120 (1995), 161-213.

[3] —, Automorphic forms with singularities on Grassmannians, ibid. 132 (1998), 491562.

[4] J. H. Bruinier and J. Funke, Traces of CM-values of modular functions, J. Reine Angew. Math. 594 (2006), 1-33.

[5] J. H. Bruinier, G. van der Geer, G. Harder and D. Zagier, The 1-2-3 of Modular Forms, Springer, 2008.

[6] J. H. Bruinier, W. Kohnen and K. Ono, The arithmetic of the values of modular functions and the divisors of modular forms, Compos. Math. 140 (2004), 552-566.

[7] D. Choi, On values of a modular form on $\Gamma_{0}(N)$, Acta Arith. 121 (2006), 299-311.

[8] D. Choi, D. Jeon, S.-Y. Kang and C. H. Kim, Traces of singular moduli of arbitrary level modular functions, Int. Math. Res. Notices 2007, art. ID rnm110, 17 pp.

[9] G. H. Hardy and E. M. Wright, An Introduction to the Theory of Numbers, Oxford Univ. Press, 1979.

[10] L. J. P. Kilford, Modular Forms: A Classical and Computational Introduction, Imperial College Press, 2008.

[11] C. H. Kim, Traces of singular values and Borcherds products, Bull. London Math. Soc. 38 (2006), 730-740.

[12] C. H. Kim and J. K. Koo, Arithmetic of the modular function $j_{1,4}$, Acta Arith. 84 (1998), 129-143.

[13] N. Koblitz, Introduction to Elliptic Curves and Modular Forms, Springer, 1993.

[14] S. P. Norton, More on moonshine, in: Computational Group Theory, Academic Press, 1984, 185-193. 
[15] D. Zagier, Traces of singular moduli, in: Motives, Polylogarithms and Hodge Theory, Part I (F. Bogomolov and L. Katzarkov (eds.), Int. Press, Somerville, 2002, 211-244.

SoYoung Choi

Department of Mathematics Education

Dongguk University

Gyeongju, 780-714, Korea

E-mail: young@dongguk.ac.kr
Chang Heon Kim

Department of Mathematics

Hanyang University

Seoul, 133-791 Korea

E-mail: chhkim@hanyang.ac.kr

Received on 1.10.2009

and in revised form on 11.3.2010 